Journal of Astronomical Instrumentation, Vol. 6, No. 2 (2017) 1740006 (10 pages)

(C) The Author(s)

DOI: $10.1142 / \mathrm{S} 2251171717400062$

\title{
Balloon Flight Demonstration of an Oscillating Heat Pipe
}

\author{
H. Fuke ${ }^{1,3}$, S. Okazaki ${ }^{1}$, H. Ogawa ${ }^{1}$ and Y. Miyazaki ${ }^{2}$ \\ ${ }^{1}$ Institute of Space and Astronautical Science \\ Japan Aerospace Exploration Agency (JAXA/ISAS) \\ Sagamihara, Kanagawa 252-5210, Japan \\ ${ }^{2}$ Thermal Control Laboratory Inc. \\ Fukui 910-0017, Japan \\ ${ }^{3}$ fuke.hideyuki@jaxa.jp
}

Received 2016 December 6; Accepted 2017 March 31; Published 2017 April 26

\begin{abstract}
The oscillating heat pipe (OHP) is a novel heat-transfer technique used in thermal engineering. Although the OHP offers many technical advantages, it has not yet been actually used in the sky. Motivated by the need to develop a cooling system for use in the balloon-borne General Anti-Particle Spectrometer (GAPS) project, we are developing OHP technologies. To demonstrate the thermal performances of an OHP in real balloon flight conditions, a scaled-down OHP model was launched by a stratospheric balloon in Japan. In this study, we report the results of the flight demonstration.
\end{abstract}

Keywords: Balloons.

\section{Introduction}

The development of the oscillating heat pipe (OHP), or pulsating heat pipe, as a cooling system has grown from the General Anti-Particle Spectrometer (GAPS) project. GAPS is an international spacescience project designed to address the dark-matter mystery, through a highly sensitive observation of cosmic-ray antiparticles (Hailey et al., 2013). GAPS will conduct this cosmic-ray observation from longduration balloon (LDB) flights over Antarctica. The first LDB science flight is planned for 2020 .

A system is being developed to cool the GAPS core detectors to their operational temperature of $238 \mathrm{~K}$ or lower, under a range of operating conditions (Fuke et al., 2016). The OHP technique will be used to transport the heat dissipated by the detectors to a separately located radiator. The OHP is a novel approach wherein heat transfer is achieved through a passive thermo-fluid-dynamics

\footnotetext{
${ }^{3}$ Corresponding author.
}

process (Akachi et al., 1996). An OHP comprises a meandering closed (or pressurized) capillary tube that passes multiple times between a heating section and a cooling section. The pressure balance between the vapor plugs and liquid slugs of the working fluid in the capillary tube excites a self-oscillating flow that transfers the heat from the heating section to the cooling section. The OHP has many advantages over a conventional heat pipe, including simple fabrication as the capillary tube needs no internal wick, less sensitivity to orientation or gravitational pull, the capacity for large-scale heat transfer, and the ability to adapt to a low heat flux. At present, the GAPS project requires heat energy of approximately $100 \mathrm{~W}$ to be transferred across a distance of more than $2 \mathrm{~m}$. This is to be achieved using an OHP with 36 turns.

The OHP is a novel technique, and has not previously been utilized in a flying vehicle such as a balloon-craft or satellite. Before moving to full-scale deployment (Okazaki et al., 2014), a flight

This is an Open Access article published by World Scientific Publishing Company. It is distributed under the terms of the Creative Commons Attribution 4.0 (CC-BY) License. Further distribution of this work is permitted, provided the original work is properly cited. 
demonstration has been conducted by mounting a scaled-down OHP as a part of the payload of an engineering balloon experiment called "pGAPS" (prototype-GAPS) (Fuke et al., 2014; Mognet et al., 2014). Initial reports of this OHP flight demonstration were presented by Fuke et al. (2012) and Okazaki et al. (2012). Brief overviews of the results have been reported in Mognet et al. (2014) and Fuke et al. (2016). In this study, we present details of the balloon-flight demonstration of the "pGAPS OHP" that have not been reported previously.

\section{Scaled-Down Oscillating-Heat-Pipe Model}

\subsection{System design}

Figure 1(a) shows an image of the scaled-down pGAPS OHP model. To show the bare pipes, this image was obtained before the cooling section was painted and the thermal insulators were attached. Figure 1(b) shows a conceptual diagram of the pGAPS OHP. This OHP had a "U-shaped" structure wherein the two vertical sides were the heating and cooling sections, and the horizontal part was the adiabatic section. The heating, adiabatic, and cooling sections were $0.3 \mathrm{~m}, 0.3 \mathrm{~m}$, and $0.4 \mathrm{~m}$ long, respectively. Dummy heater load strips were attached to the heating section, to simulate the heat dissipation of the detectors as they were cooled. The heating section was surrounded by Styrofoam insulators to suppress heat leakage. The adiabatic section was also insulated to allow the heat transport to be evaluated. The cooling section acted as a radiator dissipating the heat transported from the heating section into space by radiation. The U-shape was selected to minimize the pipework interrupting the upward view of the detectors. It should be noted that this kind of three-dimensional OHP routing with multiple turns is unique, because previously developed OHPs had planar structures (Zhang \& Faghri, 2008). An albedo reflector was attached to the backside of the adiabatic section to suppress the heat inputs to the radiator arising from the earth, in both the visible and infrared bands.

The main body of the OHP was a unicursal closed pipe with ten turns. Each turn had a check valve (Iwata et al., 2012) in the adiabatic section near the bottom of the heating section, which enhanced the performance of the OHP by directing the inner flow (Miyazaki et al., 2000). The pipes were made mainly from copper, though the points at which the check valves were mounted were made from stainless steel. To induce a gas-liquid slug flow with efficient heat transfer, all pipes had an inner diameter of $1.0 \mathrm{~mm}$. Pipes in the cooling and heating sections were connected to pipes in the adiabatic section by joint connectors. In every section, pipes were aligned to achieve the minimum bending, giving a curvature radius of $10 \mathrm{~mm}$. All pipes had a wall thickness of $0.3 \mathrm{~mm}$, designed to withstand the inner pressure of the working fluid. To allow the OHP to operate at ambient room temperatures before the flight as well as at low temperatures around $233 \mathrm{~K}$ during the flight, the refrigerant

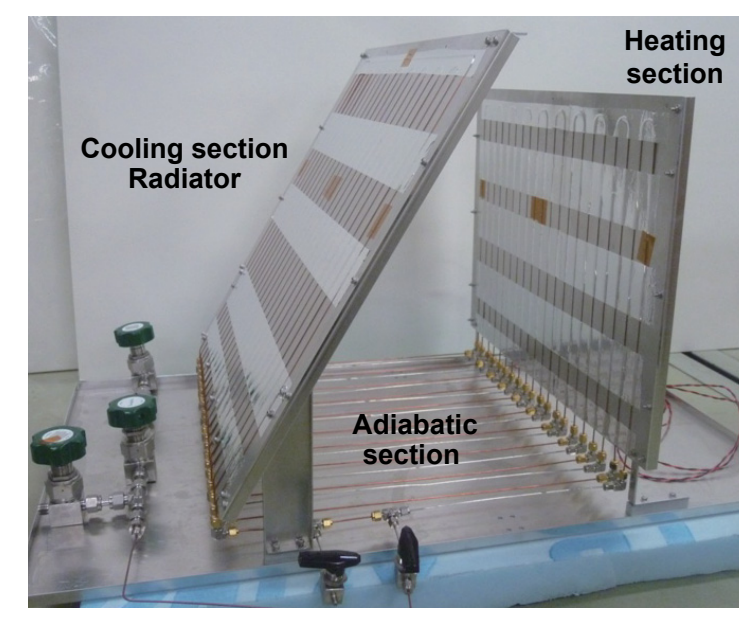

(a)

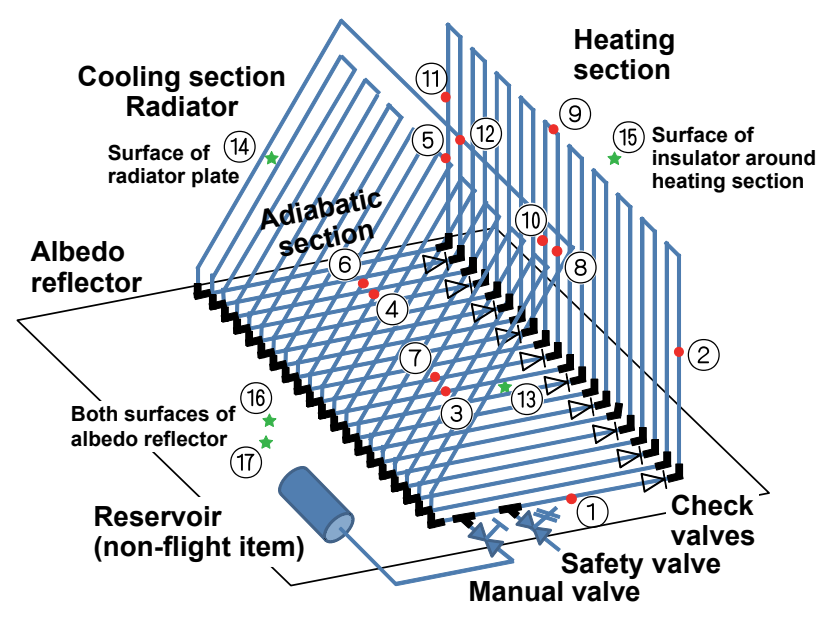

(b)

Fig. 1. (a) Photograph of the pGAPS OHP model. (b) Conceptual diagram of the pGAPS OHP. Red points (1)-(12) and green stars (13)-(17) show the attachment points of thermocouples before and after the thermal insulation was added, respectively. 
R410A was selected as the working fluid (Fuke et al., 2016). R410A is a hydrofluorocarbon (an alternative to a chlorofluorocarbon) which is a $50 \%$ / $50 \%$ mixture of difluoromethane (R32) and pentafluoroethane (R125). R410A has a moderate saturation vapor pressure in the temperature range between $220 \mathrm{~K}$ and $300 \mathrm{~K}$. Because R410A is not hazardous, it is suitable for use in a balloon experiment. Prior to the pGAPS flight, ground tests using a reservoir were conducted to determine the optimum filling ratio for operation across the wide temperature range. This was defined as the ratio between the volume of working-fluid liquid-phase in the OHP and the total volume of the OHP. To simplify the flight test, the reservoir was detached before the flight and the filling ratio was fixed at $61 \%$ (at $293 \mathrm{~K}$ equivalent).

Based on the thermal model described below, we estimated that no OHP section would be exposed to temperatures exceeding $343 \mathrm{~K}$ in the course of the balloon flight. The saturated vapor pressure of R410A is $4.8 \mathrm{MPa}$ at $343 \mathrm{~K}$ (JSTP, 2008), and a pressurization test confirmed that the pGAPS OHP was able to withstand an inner pressure exceeding $15 \mathrm{MPa}$, giving a safety factor greater than three. To ensure mechanical safety, a pressure release valve with a release pressure set at $10 \mathrm{MPa}$ was installed. To further prevent an overpressure arising, thermostatic switches were added to the powersupply lines. These would automatically disconnect the power supply to the heater load if the temperature in the heating section exceeded $343 \mathrm{~K}$. A manual release valve was also added, to allow emergency release before the flight or after the recovery of the payload. Leak tests were conducted before the flight. These confirmed that the leakage of working fluid was negligibly small across the design temperature range.

\subsection{Thermal properties}

To maximize thermal contact between the cooling pipes and the radiator, semi-circular grooves were cut into the aluminum plate of the radiator and the cooling pipes were placed within them. Dedicated tests were conducted to select a commercially available thermal adhesive to fill the gap between the pipes and the grooves. A two-part adhesive made from micronized silver (TA-01; Arctic Silver Inc.) was selected. This has a thermal conductivity greater than $7.5 \mathrm{~W} / \mathrm{m} \cdot \mathrm{K}$, can tolerate the $203 \mathrm{~K}$ temperature at the tropopause, and is relatively easy to handle.

The radiator was painted white to ensure low solar absorptance $\left(\alpha_{\mathrm{s}}\right)$ and high infrared emissivity $\left(\epsilon_{\mathrm{n}}\right)$. A commercially available white paint was selected also based on dedicated evaluation tests, with three criteria: (i) the capacity to remain attached to both aluminum and copper surfaces at temperatures as low as $203 \mathrm{~K}$. (ii) a low $\alpha_{\mathrm{s}}$ and a high $\epsilon_{\mathrm{n}}$. (iii) ease of handling to survive a balloon experiment. The painting procedure was as follows: the pipe surface was first polished using sandpaper and the radiator surface given a chromate conversion coating. The surface was then coated with a primer (Metal Primer Spray; Asahipen Corp.), and the paint layer was added (Water-based Versatile Spray; Asahipen Corp.). The painted surface had an $\alpha_{\mathrm{s}}$ of 0.277 and an $\epsilon_{\mathrm{n}}$ of 0.924 . We measured the $\alpha_{\mathrm{s}}$ using a spectral photometer (U-4100; Hitachi Ltd.), and the $\epsilon_{\mathrm{n}}$ (normal emittance) using an emissometer/reflectometer (TESA 2000; AZ Technology Corp.).

Prior to flight, the thermal performance of the pGAPS OHP was evaluated by testing in a thermal vacuum chamber to simulate both the temperature and pressure of the ambient atmosphere during the flight. We confirmed that the pGAPS OHP was able to operate across a temperature range from $220 \mathrm{~K}$ to $300 \mathrm{~K}$. A heat conductance of around $5 \mathrm{~W} / \mathrm{K}$ was achieved throughout this temperature range, capable of transporting a heat load of around $10 \mathrm{~W}$. The heat conductance was defined as the temperature difference between the heating and cooling sections, divided by the amount of heat transported. If the working fluid does not contribute to heat transport, or if the pGAPS OHP fails to function, the heat conductance of the pGAPS OHP would decrease to the conductance of the pipes themselves, or around $0.002 \mathrm{~W} / \mathrm{K}$. The operation of the OHP could therefore be measured from its heat conductance. For reference, the maximum amount of heat transportable by this OHP was approximately $100 \mathrm{~W}$ at $293 \mathrm{~K}$.

\subsection{Mounting as payload}

Figure 2(a) shows the pGAPS OHP mounted as payload. To ensure that the evaluation was standalone, the OHP was isolated both thermally and electrically from the other payload components, and from the payload support structure. 

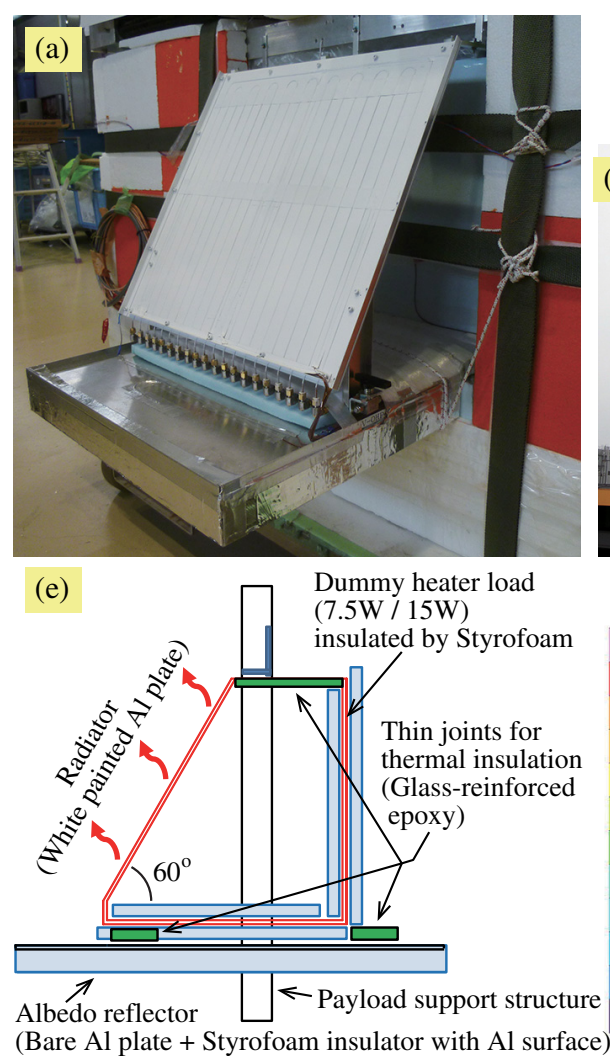

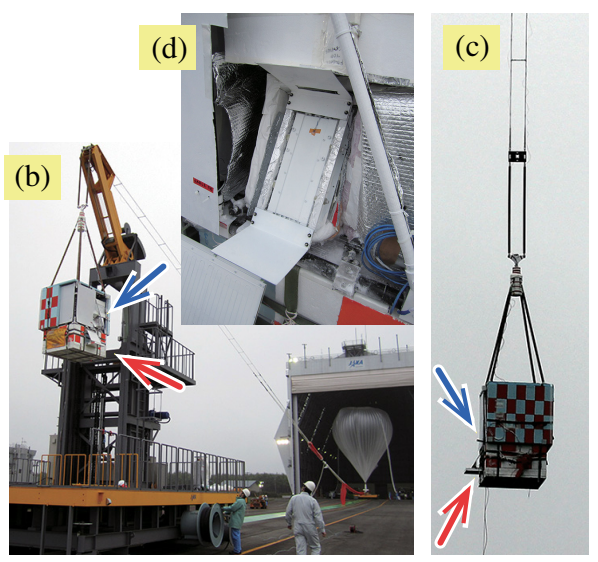

(f)

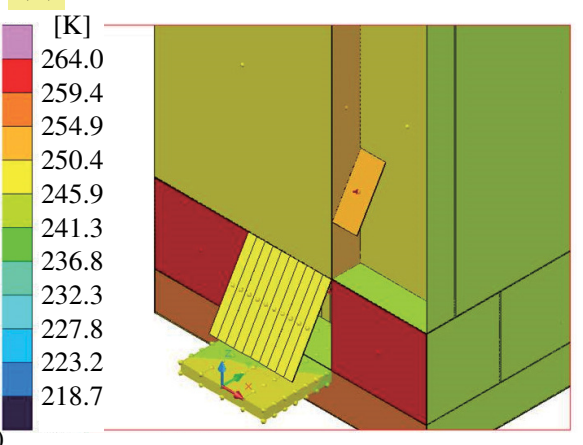

Fig. 2. (a) Scaled-down OHP model mounted as the payload. (b) pGAPS payload before launch. (c) pGAPS payload immediately after launch. Red and blue arrows in (b) and (c) indicate the OHP radiator and pGAPS cooling radiator, respectively. (d) pGAPS cooling radiator. (e) Conceptual diagram of the thermal insulation of the pGAPS OHP. (f) Thermal model of the pGAPS payload.

As can be seen from Fig. 2(e), the OHP was connected to the payload structure by thin joints made from glass-reinforced epoxy (GRE). The total heat conductance of these GRE joints was as little as $0.02 \mathrm{~W} / \mathrm{K}$.

As shown in Fig. 1(b), thermocouples were attached at 17 points on the pGAPS OHP. Type T (copper-constantan) thermocouples were used. The red points (1)-(12) and the green stars (13)-(17) show the attachment points before and after the thermal insulation was added. Thermocouples (13) and (15) were attached to the surface of the insulator around the adiabatic and heating sections, respectively. Thermocouple (14) was attached to the white-painted radiator surface. Thermocouples (16) and (17) were attached to the top and bottom surface of the albedo reflector, respectively. A dedicated standalone data logger was mounted to record flight data. Temperature data was stored by the logger at a rate of $1 \mathrm{~Hz}$.

A dedicated receiver was mounted to receive radio commands from the ground station. This allowed the status of the dummy heater to be switched between three modes: (i) no load (switched off), (ii) a $7.5 \mathrm{~W}$ load, and (iii) a $15 \mathrm{~W}$ load. These were selected to allow the OHP to be operated at two kinds of modest heat load, while minimizing the weight of the batteries needed. The precise heat load was monitored and recorded by the onboard logger.

As shown in Fig. 2(d), a second radiator was mounted on the pGAPS payload to dissipate the heat generated by a subsystem. To distinguish this from the OHP radiator, we named this the pGAPS cooling radiator. Because it was on the same azimuth as the OHP radiator but thermally decoupled from it, the pGAPS cooling radiator provided a reference for use in the post-flight thermal analysis.

\subsection{Thermal calculation model}

A thermal analysis was conducted, using the finite element method (FEM) software Thermal Desktop (C \& R Technologies Inc.). Figure 2(f) gives an example of the simulated temperatures of the OHP, the pGAPS cooling radiator, and the payload sidewalls. Using this thermal model, an optimal tilt 
angle of $30^{\circ}$ from the vertical was determined for the OHP radiator. This tilt angle maximized the view factor of the OHP radiator toward space, while minimizing the heat inputs both from the earth and from the solar radiation reflected by the payload sidewalls and the balloon. The mechanical dimensions of the albedo reflector, including its area and thickness, were also optimized using this thermal model. The upper surface of the aluminum albedo reflector was left bare, with no coating, to minimize the reflected solar-radiation inputs to the OHP radiator. The back side of the albedo reflector carried a Styrofoam insulator with an aluminized surface to suppress the heat inputs from the earth. Because no explicit theory is available to support simulations of the OHP performance, we used the OHP heat conductance in the thermal model to approximate $\mathrm{OHP}$ heat transport. The pGAPS cooling radiator was also coded into the thermal model for use as a reference. To simplify the overall calculations, the FEM model of the pGAPS cooling radiator was less complex than that of the OHP radiator. Based on this thermal model, we predicted that the OHP radiator would reach a temperature of $238 \mathrm{~K}$, on the assumption that it was kept oriented to the anti-sun azimuth at an altitude of $35 \mathrm{~km}$.

\section{Flight Operation}

The pGAPS flight was conducted as an engineering test flight in the GAPS program. The test flight was designed to demonstrate the basic performance of each GAPS subsystem (including the OHP) in real flight conditions.

The pGAPS payload was launched at 04:55 local time on June 3rd 2012 by the Japan Aerospace Exploration Agency (JAXA), from a balloon base at the Taiki Aerospace Research Field (TARF) in Japan (Fuke et al., 2010). Figures 2(b) and 2(c) show the pGAPS payload before and immediately after launch. The red and blue arrows identify the OHP radiator and the pGAPS cooling radiator, respectively.

After a 3-h ascent, of which $1.5 \mathrm{~h}$ were spent drifting eastward according to the so-called boomerang flight operation (Nishimura \& Hirosawa, 1981), the balloon reached its floating (or level) altitude at 08:05. It was then carried westward for $3 \mathrm{~h}$ at the floating altitudes around $31-33 \mathrm{~km}$. The flight was terminated near the coast, and the payload was safely recovered from the sea, as planned.
At launch, the heater load in the heating section was kept switched off. At 05:40, shortly after the start of the boomerang operation, the heater was turned on with a load of $15 \mathrm{~W}$. From 06:42 AM to 08:11, during the balloon re-ascent, the heater load was switched to $7.5 \mathrm{~W}$. During the balloon floating, in the period to $09: 43$, the load was returned to $15 \mathrm{~W}$, then shifted back to $7.5 \mathrm{~W}$ before being switched off shortly before the flight termination. All of these switches were controlled from the ground station, as planned. The flight data was successfully recorded by the onboard logger.

The original plan was to direct the radiators to the anti-sun azimuth during the floating period. However, owing to an operational mishap, the payload lost azimuth control. As a result, the radiators were pointed to the sunny side throughout the first $40 \mathrm{~min}$ of this period, after which the payload rotated around its vertical axis.

\section{Flight-Data Analysis}

\subsection{Heat conductance}

The red, blue, and green lines in Fig. 3(a) show the in-flight temperature data from the OHP heating section, the OHP cooling section, and the OHP albedo reflector, respectively. Minute-averaged data points from representative thermocouples are plotted. Cyclic variations in temperature, with a period of several minutes, reflect the rotation of the payload. Below Fig. 3(a), periods of the boomerang, reascent, and floating operations are indicated by orange arrows, and mode switching of the heater load is shown by red arrows.

The sky-blue dots in Fig. 3(b) show the calculated heat conductance. The heat conductance was simply defined as the ratio of the heater load divided by the temperature difference between the heating and cooling sections. Due to the rotation of the payload, the OHP did not reach complete thermal equilibrium. We therefore used data from the periods at which the time variation in the temperature gradient between the heating and cooling sections was minimal. The sky-blue dots show the heat conductance obtained from this quasi-stable data. The heat conductance was maintained at around $5 \mathrm{~W} / \mathrm{K}$ and was significantly greater than $0.002 \mathrm{~W} /$ $\mathrm{K}$ throughout the flight, as expected. The results confirmed that the pGAPS OHP could be used in a balloon flight. 


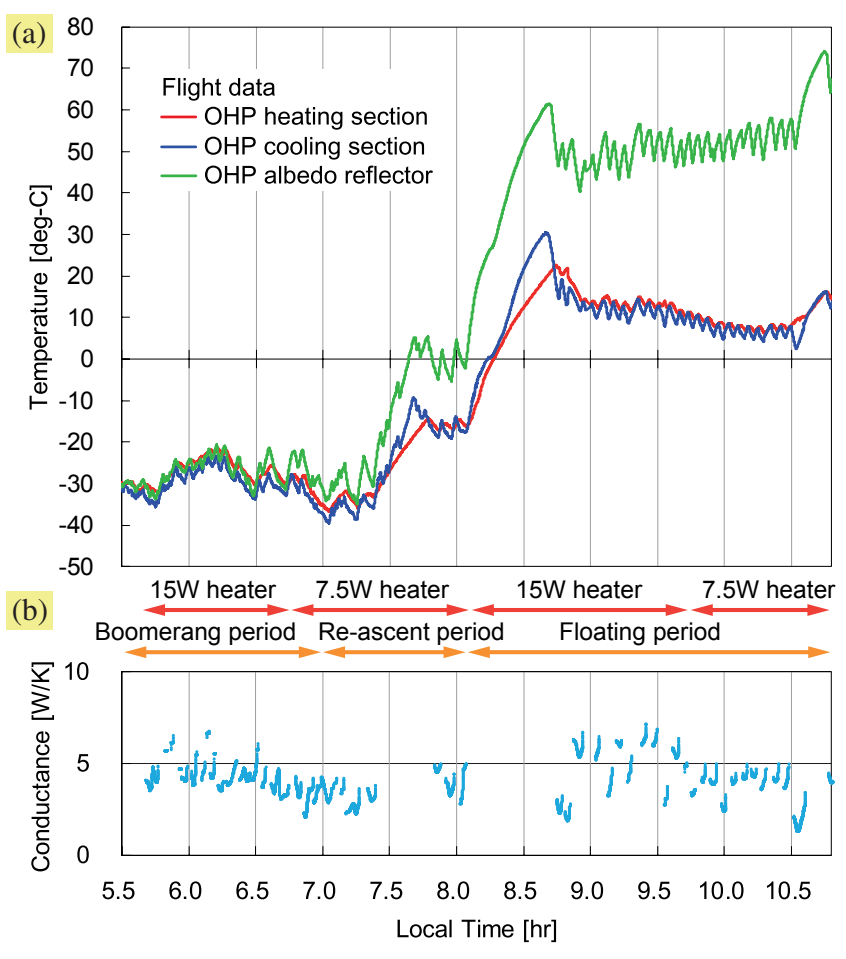

Fig. 3. (a) Red, blue, and green lines show temperatures measured during flight at the OHP heating section, the OHP cooling section, and the OHP albedo reflector, respectively. (b) Sky-blue dots show the OHP heat conductance when the temperature gradient in the OHP was quasi-stable. Periods of the boomerang, re-ascent, and floating operations are indicated by orange arrows. Mode switching of the heater load is shown by red arrows.

\subsection{Setting variables in thermal model}

To confirm the validity of the OHP flight data in more detail, we conducted a transient temperature simulation, using the thermal model and thermal properties discussed in Sec. 2.

During the flight, the three-dimensional location of the payload was monitored by a global positioning system (GPS). The purple dashed line in Fig. 4 shows the GPS altitude data. Heat inputs from solar radiation at each moment were estimated from the relative orientation of the payload, using in-flight attitude data collected by a gyro and a solar sensor. The ambient pressure $\rho(x)$ and ambient temperature $T(x)$ at each altitude were approximated by Eqs. (1) and (2), respectively, as functions of the altitude $x$. These equations were obtained by fitting to tables of the U.S. standard atmosphere (NOAA, 1976) as shown in Fig. 5.

$$
\rho(x)=2.035 e^{-0.157 x} .
$$

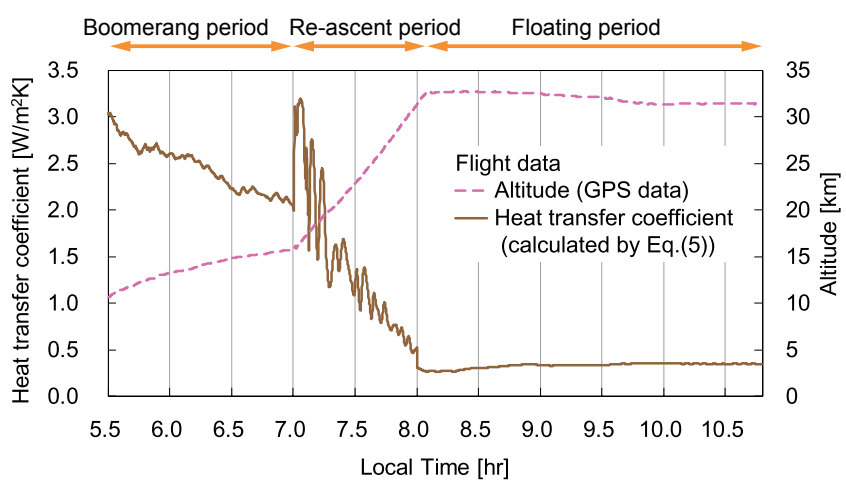

Fig. 4. The purple dashed line shows the GPS altitude data. The brown line shows the estimated convective heat transfer coefficients used in the thermal calculation. Periods of the boomerang, re-ascent, and floating operations are indicated by orange arrows (the same as those in Fig. 3).

$$
T(x)=0.604 x^{2}-2.109 x+233.53 .
$$

Thermal conductivity in rarefied atmosphere is not yet well understood theoretically. In this work, heat convection from the ambient atmosphere during the balloon re-ascent period was treated as forced convection. In contrast, heat convection in both the boomerang operation at altitudes of around $15 \mathrm{~km}$, and the floating period at approximately $32 \mathrm{~km}$, was treated as natural convection in the vertical direction. The Nusselt numbers, $N_{u}$, of the vertical natural convection and forced convection were estimated by Eqs. (3) and (4), respectively (JSME, 2009).

$$
N_{u}=s_{1} \frac{0.508 P_{r}^{1 / 2} G_{r}^{1 / 4}}{\left(0.952+P_{r}\right)^{1 / 4}},
$$

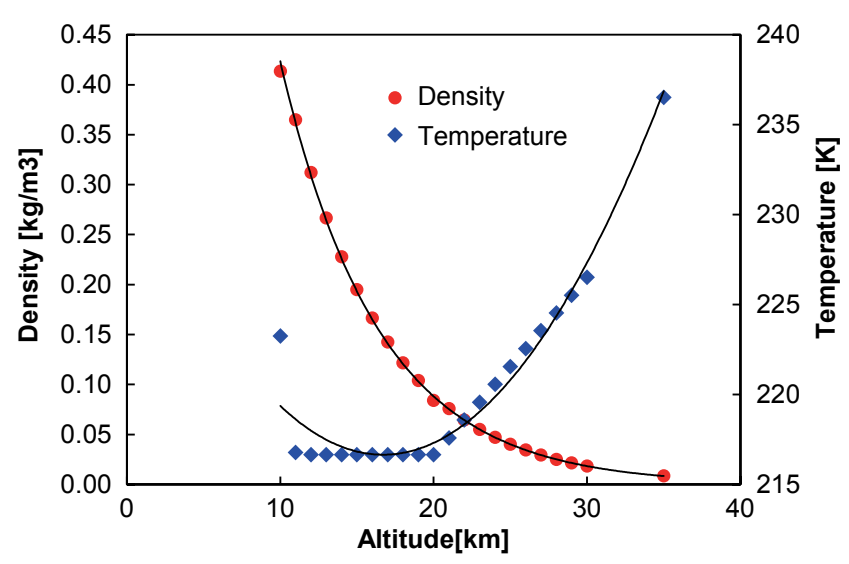

Fig. 5. Red dots and blue diamonds show the altitude dependence of the density and temperature of the U.S. standard atmosphere. The two solid lines show Eqs. (1) and (2) fitted to the data. 


$$
N_{u}=s_{2} 0.037 P_{r}^{1 / 3} R_{e}^{4 / 5} .
$$

Here, $P_{r}, G_{r}$, and $R_{e}$ denote the Prandtl number, the Grashof Number, and the Reynolds number, respectively. In the calculation of $R_{e}$, fluid velocities relative to the payload were approximated from the balloon ascent velocities, monitored by a GPS. The rotation of the payload was not taken into account in the estimation of $N_{u}$. Scaling parameters $s_{1}$ and $s_{2}$, which cannot be determined theoretically, were estimated from global time-variable trends of temperature data shown in Fig. 3(a). For the floating period, $s_{1}$ was set at the standard value of 1 . For the boomerang period, $s_{1}$ was assumed to be increased due to the gradual ascent of the balloon and was set to 2 . For the re-ascent period, $s_{2}$ was set to $2 / 3$, which indicates the ratio of laminar flow to turbulent flow assumed in Eq. (4) is to be slightly modified in this case. Given the uncertainty of the $N_{u}$ estimation, these modified values of $s_{1}$ and $s_{2}$ were quite close to unity.

The convective heat transfer coefficient, $h$, was calculated by Eq. (5) (JSME, 2009).

$$
h=\lambda N_{u} / d \text {. }
$$

Here, the representative length, $d$, was set at $0.42 \mathrm{~m}$, the length of the OHP radiator panel. The thermal conductivity of air, $\lambda$, was set to $0.0202 \mathrm{~W} /$ $\mathrm{m} \cdot \mathrm{K}$ which was taken from the corresponding table of the U.S. standard atmosphere at the typical ambient temperature of $223 \mathrm{~K}$. The brown line in Fig. 4 shows the calculated $h$. Periodic variations in $h$ during the re-ascent period represent fluctuations in the ascent speed of the balloon.

All material properties, such as the heat capacity of the OHP components and the thermal resistance of the insulators, were taken into account, based on the references (Gilmore, 2002; JSTP, 2008). The calculated total heat capacity of the pGAPS OHP was about $1.65 \mathrm{~kJ} / \mathrm{K}$. The $\alpha_{\mathrm{s}}$ and the $\epsilon_{\mathrm{n}}$ of the white painted surface was set at measured values of 0.277 and 0.924 , respectively, as discussed in Sec. 2.2. Only the $\alpha_{\mathrm{s}}$ and the $\epsilon_{\mathrm{n}}$ of the aluminum surface (both bare aluminum surface and aluminized surface) of the albedo reflector were modified. An aluminum surface becomes naturally oxidized, and the albedo reflector was handled many times during the launch preparation. This changed the surface properties from their standard reference values (JSTP, 2008). By adjusting to the flight data of the OHP albedo reflector temperature, the $\alpha_{\mathrm{s}}$ and the $\epsilon_{\mathrm{n}}$ of the aluminum surface were set to 0.28 and
0.18 , respectively, rather than their standard values of 0.15 and 0.05 . Because all the components except for the OHP albedo reflector had no aluminum surface, these modifications had no major influence on the temperature calculations for other components. In the future, the aluminum surface on the GAPS science gondola to be developed must be treated more carefully to suppress the variation of its surface properties.

In the thermal model, the heat conductance of the OHP was assumed to be a constant $5 \mathrm{~W} / \mathrm{K}$ which was a nominal value measured before the flight and was consistent with the flight data as discussed in Sec. 4.1.

As a summary of this section, almost all variables used in the thermal calculation model were set to measured values or referenced numbers as planned before the flight. Modifications were added only to the following uncertain thermal properties: the $\alpha_{\mathrm{s}}$ and the $\epsilon_{\mathrm{n}}$ of the aluminum surface and the $N_{u}$ for the boomerang and re-ascent periods. These modifications could change the calculated temperatures of the OHP heating and cooling sections only a few $\mathrm{K}$ and could not overturn the validity of the thermal model discussed in the next section.

\subsection{Thermal calculation results}

Implementation of these calculations in the FEM thermal model gave simulated temperature profiles of the pGAPS payload during the boomerang, re-ascent, and floating periods.

The red, blue, and green solid lines in Fig. 6(a) are the same as those shown in Fig. 3(a) (in-flight temperature data from the OHP heating section, the OHP cooling section, and the OHP albedo reflector, respectively). The black solid line in Fig. 6(a) shows the temperature measured at the pGAPS cooling radiator.

The red, blue, green, and black dashed lines in Fig. 6(a) show the simulated temperatures. They were in good agreement with the flight data shown by solid lines of the same color. Even the simplified pGAPS cooling radiator was well simulated by the thermal model. Figures 6(b) and 6(c) show enlargements of the temperatures at the heating and cooling sections in Fig. 6(a) from the boomerang and floating periods, respectively. It can be seen that the temperature variations of the OHP heating and cooling sections were replicated very accurately. Small temperature deviations between the flight 


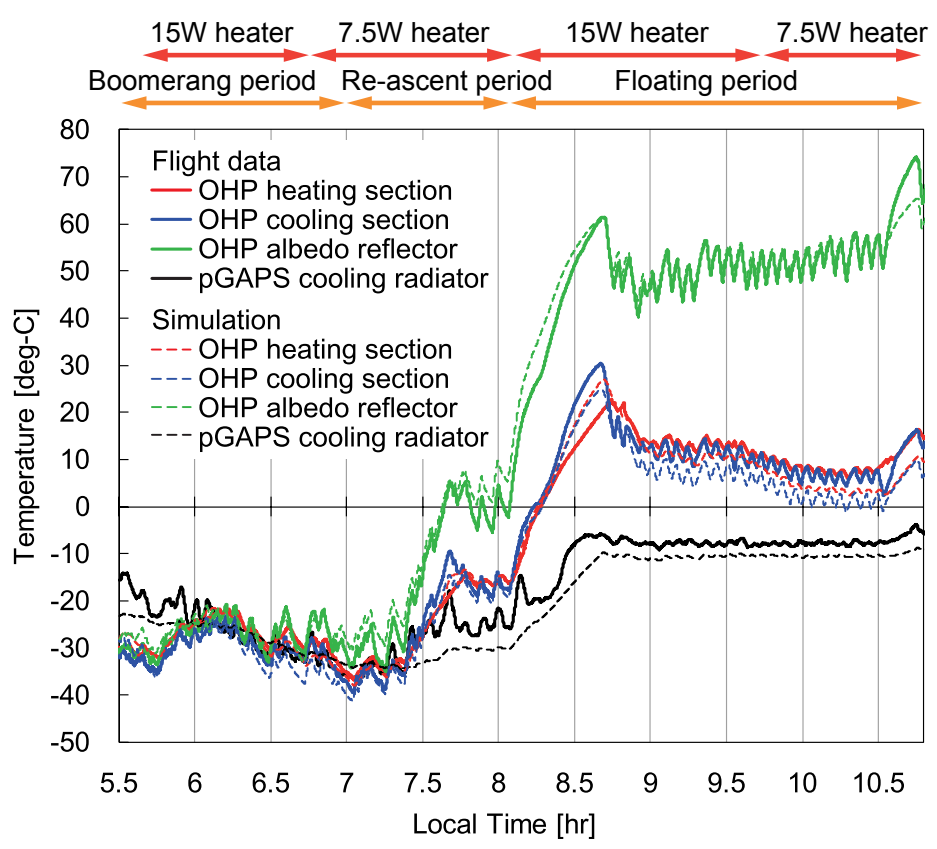

(a)

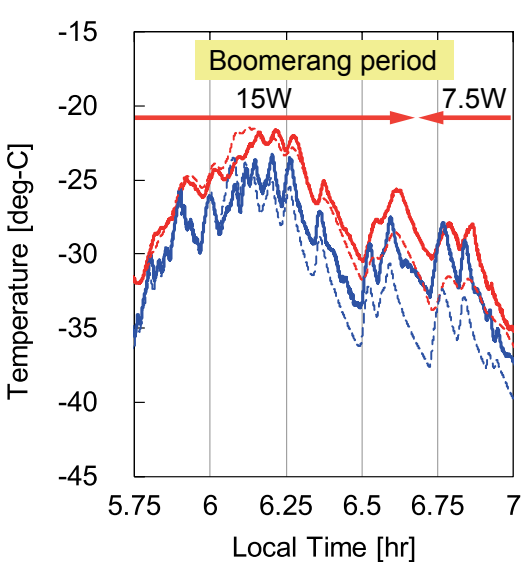

(b)

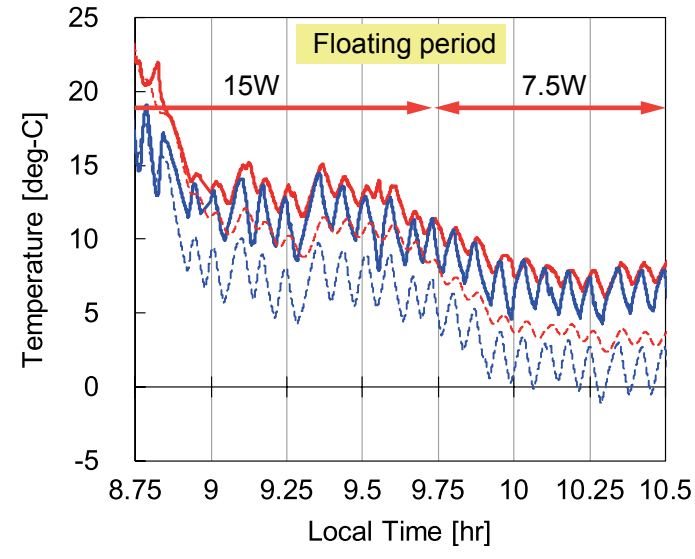

(c)

Fig. 6. (a) Red, blue, green, and black solid lines show temperatures measured during flight at the OHP heating section, the OHP cooling section, the OHP albedo reflector, and the pGAPS cooling radiator, respectively. The red, blue, and green curves are the same as those shown in Fig. 3. Temperatures calculated by the thermal model, shown by dashed lines of each color, closely matched the flight data. Figures (b) and (c) are partial enlargements of Fig. (a). In the same manner as shown in Fig. 3, balloon flight operations and heater-load mode switching are shown by orange and red arrows, respectively.

data and the simulated data were assumed to be mainly due to uncertainties in the environmental conditions, such as temperature differences between the U.S. standard and real atmosphere.

As a case study, temperatures were simulated on the assumption that the OHP did not function at all (or the OHP heat conductance was $0.002 \mathrm{~W} / \mathrm{K}$ ). The red and blue dashed-dotted lines in Fig. 7 show the simulated temperatures of the heating and cooling sections, respectively. The equivalent temperatures for the OHP albedo reflector and the pGAPS cooling radiator are not shown in Fig. 7, because they are less dependent on OHP conductance. The solid and dashed lines in Fig. 7 are the same as those shown in Fig. 6. It is clear from the divergence between the simulated temperatures and the flight data that the simulation of a functionless OHP failed to model the real values. This result reconfirmed that the pGAPS OHP operated successfully throughout the flight. 


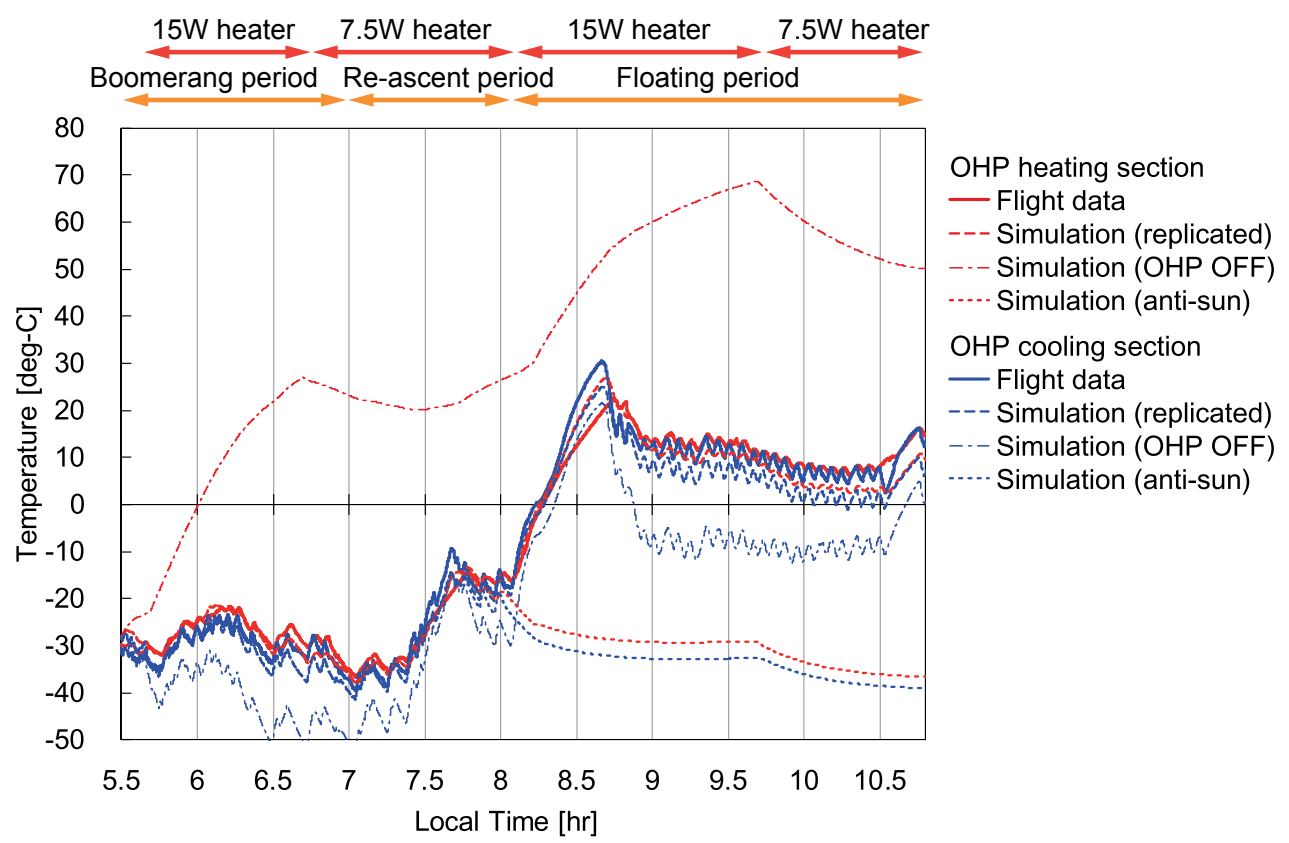

Fig. 7. Solid and dashed lines are the same as those shown in Fig. 6 (Red and blue lines represent temperatures at the OHP heating and cooling sections. Solid and dashed lines show temperatures measured during flight and replicated by the simulation, respectively). Dashed-dotted lines show the simulated temperatures assuming a functionless OHP. Divergence of these dashed-dotted lines from solid lines confirms that the OHP operated during flight. Dotted lines show the simulated temperatures on the assumption that the OHP cooling section was pointed to the anti-sun direction during the floating period. In the same manner as shown in Fig. 6 , balloon flight operations and heater-load mode switching are shown by orange and red arrows, respectively.

As another case study, temperatures were simulated on the assumption that the radiators were pointed to the anti-sun azimuth during the floating period as planned. The red and blue dotted lines in Fig. 7 show the simulated temperatures of the heating and cooling sections, respectively. The simulated temperatures indicated that the temperature of the OHP heating section should reach about $238 \mathrm{~K}$ during the float period as predicted in Sec. 2.4 .

All these simulated results confirmed the validity of the thermal model which was used to design the pGAPS OHP. The loss of the payload azimuthal control turned out well because it provided us an opportunity to verify the thermal model in an unexpectedly wide temperature range and under unexpected transient conditions. The thermal model will be applied in designing the GAPS science gondola including the full-scale OHP.

\section{Summary}

We developed a novel U-shaped OHP with 10 turns, each of approximately $1 \mathrm{~m}$ in length. Using R410A as the working fluid, this OHP functioned successfully across a temperature range from $220 \mathrm{~K}$ to $300 \mathrm{~K}$. The OHP was mounted as the pGAPS payload and tested in a real balloon flight. The flight data confirmed that the pGAPS OHP operated with the appropriate heat conductance. A transient temperature simulation accurately replicated the flight data, confirming the validity of the thermal model. Our results demonstrated that the pGAPS OHP functioned as expected throughout the test flight. This was the first successful demonstration of an OHP in the sky. ${ }^{(a)}$ Some of the components used, such as the white paint and the thermal adhesive, were readily available commercial products. The data generated will be useful in later balloon flights. The equations used to estimate convective heat transfer coefficients in a rarefied atmosphere will also provide a useful guideline for further balloon experiments.

\footnotetext{
${ }^{\mathrm{a}}$ For reference, a small planate OHP developed by another group was launched by a JAXA's engineering satellite "SDS-4" to conduct the first on-orbit OHP demonstration. Okamoto et al. (2013) reported that its initial operation was in July 2012, a month after our balloon experiment.
} 


\section{Acknowledgments}

We thank JAXA/ISAS for providing a scientific balloon (DAIKIKYU) flight opportunity and for their professional support in carrying out the pGAPS flight. We are grateful for the support of Y. Shibano, G. Tajiri, and all of the GAPS collaborators in implementing this work. This work was partly supported by MEXT Grants-in-aid KAKENHI (JP20740166, JP22340073, and JP26707015).

\section{References}

Akachi, H., Polasek, F. \& Stulc, P. [1996] Proc. 5th International Heat Pipe Symposium, Melbourne, p. 208.

Fuke, H., Akita, D., Iijima, I. et al. [2010] Adv. Space Res. 45, 490.

Fuke, H., Okazaki, S., Okubo, T. et al. [2012] 39th COSPAR Scientific Assembly, Mysore, PSB.1-0012-12.

Fuke, H., Ong, R. A., Aramaki, T. et al. [2014] Adv. Space Res. 53, 1432.

Fuke, H., Abe, T., Daimaru, T. et al. [2016] Trans. Jpn. Soc. Aeronaut. Space Sci. 14 (ists30), Pi_17, doi: 10.2322/ tastj.14.Pi_17.

Gilmore, D. G. (ed.) [2002] Spacecraft Thermal Control Handbook: Fundamental Technologies (Aerospace Corporation, CA, USA).
Hailey, C. J., Aramaki, T., Boggs, S. E. et al. [2013] Adv. Space Res. 51, 290.

Iwata, N., Ogawa, H. \& Miyazaki, Y. [2012] Heat Pipe Sci. Technol. An Int. J. 3(2-4), 223-231.

Japan Society of Thermophysical Properties Series (JSTP) (ed.) [2008] Thermophysical Properties Handbook (Yokendo, Tokyo Japan), pp. 446-448 (in Japanese).

Miyazaki, Y., Polasek. F. \& Akachi, H. [2000] Proc. 6th Int. Heat Pipe Symposium, Chiang Mai, pp. 389-393.

Mognet, S. A. I., Aramaki, T., Bando, N. et al. [2014] Nucl. Instrum. Methods A 735, 24.

Nishimura, J. \& Hirosawa, H. [1981] Adv. Space Res. 1, 239.

Okamoto, A., Ando, M. \& Sugita, H. [2013] Proc. 17th Int. Heat Pipe Conf., Kanpur, p. 32.

Okazaki, S., Fuke, H., Ogawa, H. et al. [2012] 42nd Int. Conf. Environmental Systems, San Diego, AIAA, pp. 2012-3499.

Okazaki, S., Fuke, H., Miyazaki, Y. et al. [2014] J. Astron. Instrum. 3(2), 1440004.

The Japan Society of Mechanical Engineers (JSME) (ed.) [2009] Heat Transfer Engineering Materials, 5th edn. (Maruzen, Tokyo Japan) (in Japanese).

The National Oceanic and Atmospheric Administration (NOAA), the National Aeronautics and Space Administration (NASA), and the U.S. Air Force (ed.) [1976] U.S. Standard Atmosphere, U.S. Government Printing Office, Washington, D.C.

Zhang, Y. \& Faghri, A. [2008] Heat Transfer Eng. 29(1), 20-44. 